\title{
Multivessel spontaneous coronary artery dissection presenting as acute myocardial infarction
}

\author{
Seung Ki Moon, Seung Uk Lee, Dong Hoon Yang, Cheol Min Bak, Shin Hyoung Jo, Sung Soo Kim, \\ Dong Goo Kang, and Sang Ki Cho
}

Division of Cardiology, Department of Internal Medicine, Kwangju Christian Hospital, Gwangju, Korea

Received: August 25, 2015 Revised : September 30, 2015 Accepted: December 22, 2015

\author{
Correspondence to \\ Seung Uk Lee, M.D. \\ Division of Cardiology, Depart- \\ ment of Internal Medicine, \\ Kwangju Christian Hospital, 37 \\ Yangnim-ro, Nam-gu, Gwangju \\ 61661, Korea \\ Tel: $+82-62-650-5230$ \\ Fax: +82-62-650-5116 \\ E-mail: cardiosu@hanmail.net
}

To the Editor,

Spontaneous coronary artery dissection $(\mathrm{SCAD})$ is a very rare cause of acute myocardial ischemia associated with a high mortality rate [1]. The first angiographic diagnosis was reported almost 50 years. Since then, approximately 700 cases of SCAD have been reported [2,3]. Its incidence, etiology, and pathogenesis remain unclear. SCAD is most common in young people, particularly in peripartum women without traditional risk factors; however, it can also occur in elderly patients with atherosclerosis. The clinical presentation depends on the extent and flow-limiting severity of the coronary dissection, and ranges from asymptomatic to sudden cardiac death. There are no specific guidelines on how to manage patients with SCAD. This report describes an elderly man with multivessel SCAD involving the right coronary artery (RCA) and left anterior descending artery (LAD) presenting as acute myocardial infarction, which was successfully treated with percutaneous coronary intervention.

A 63-year-old man was transferred to the emergency department complaining of squeezing chest pain that had increased over the past 4 days. He had no risk factors for coronary artery disease and no history of trauma, infection, or systemic vasculitis. Physical examination showed a blood pressure of $120 / 70$
$\mathrm{mmHg}$ and a regular heart rate of 66 beats per minute, with normal $\mathrm{S} 1$ and S2 sounds. On auscultation, his lungs were clear. The initial electrocardiogram (ECG) showed premature ventricular beats and the cardiac enzymes were elevated (CK 314 U/L [range, 43 to 165], CK-MB $38.46 \mathrm{ng} / \mathrm{mL}$ [range, o to 3.61 ], troponin-I $0.96 \mathrm{ng} / \mathrm{mL}$ [range, o to 0.16 ], and troponin $\mathrm{T} 0.071 \mathrm{ng} / \mathrm{mL}$ [range, o to o.014]). Transthoracic echocardiography showed akinesia of the inferior and septal walls with a decreased left ventricular ejection fraction (EF, 40\%). He underwent emergency coronary angiography, which revealed a long, diffuse lesion from the proximal to mid RCA and from the proximal to mid LAD, with a suspicious radiolucent intimal flap (Fig. 1). Suspecting SCAD, intravascular ultrasound (IVUS, Boston Scientific, Valencia, CA, USA) was performed. This showed dissecting flaps with intramural hematomas compressing the true lumen (Fig. 2). No atherosclerotic changes were detected in either vessel on IVUS. The ongoing chest discomfort and elevated cardiac enzymes suggested primary revascularization because the coronary dissection involved the proximal LAD and RCA. He was treated with everolimus-eluting stents (XIENCE Xpedition, Abbott Vascular, Santa Clara, CA, USA) in the proximal-medial RCA and LAD (proximal RCA, $4.0 \times 33 \mathrm{~mm}$; 


\section{KJIM}

The Korean Journal of Internal Medicine Vol. 33, No. 3, May 2018

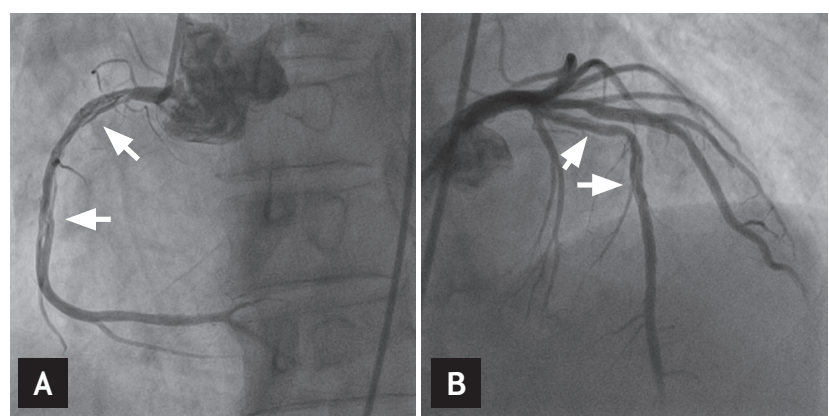

Figure 1. Coronary angiography showed long, diffuse lesions extending from the proximal to middle right coronary artery (A) and proximal to middle left anterior descending artery (B) with a suspicious radiolucent intimal flap (arrows).

middle RCA, $4.0 \times 38 \mathrm{~mm}$; proximal LAD, $4.0 \times 14 \mathrm{~mm}$; and middle LAD, $3.5 \times 38 \mathrm{~mm}$ ) (Fig. 3). Subsequent IVUS showed a fully covered dissection lesion and the final angiogram showed good distal flow without residual stenosis. After an uneventful recovery, he was discharged on dual antiplatelet therapy and has been followed as an outpatient. At the 12-month follow-up, the patient was well and asymptomatic.

To the best of our knowledge, this report is the first report of multivessel SCAD diagnosed using IVUS and successfully treated with percutaneous intervention (PCI) in Korea. Our patient had SCAD in both the LAD and RCA without underlying coronary risk factors or other coronary anomalies, such as fibromuscular dysplasia (FMD).

SCAD is a rare disease that occurs most commonly in young people, particularly in peripartum or postpartum women without traditional risk factors for coronary artery disease. SCAD is found in $0.2 \%$ to $1.0 \%$ of patients who undergo angiography for acute coronary syndrome [4]. The majority of SCAD cases involve a single vessel, usually the LAD. Multivessel SCAD, such as our case, is extremely rare and only a few cases have been reported. Furthermore, most reported multivessel SCAD cases are related in closely vessels.

SCAD is defined as a nontraumatic, noniatrogenic separation of the coronary artery walls, creating a false lumen [3]. The first of two proposed mechanisms involves initiation of dissection and hemorrhage by an intimal tear and creation of a false lumen. The second mechanism is rupture of the vasa vasorum, leading to intramural hemorrhage and medical dissection without intimal tearing. In many situations, SCAD is poorly characterized by angiography, as the narrowing caused by the intramu-

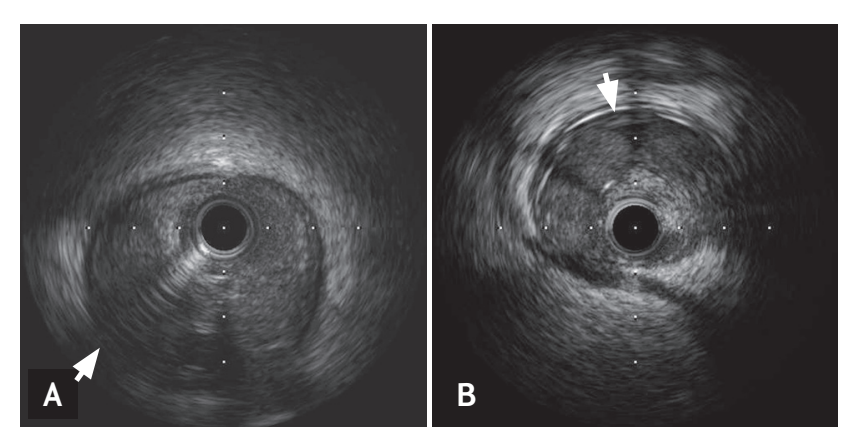

Figure 2. Intravascular ultrasound confirmed the dissecting flaps with intramural hematomas compressing the true lumens of the proximal right coronary artery (A, arrow) and proximal left anterior descending artery (B, arrow).
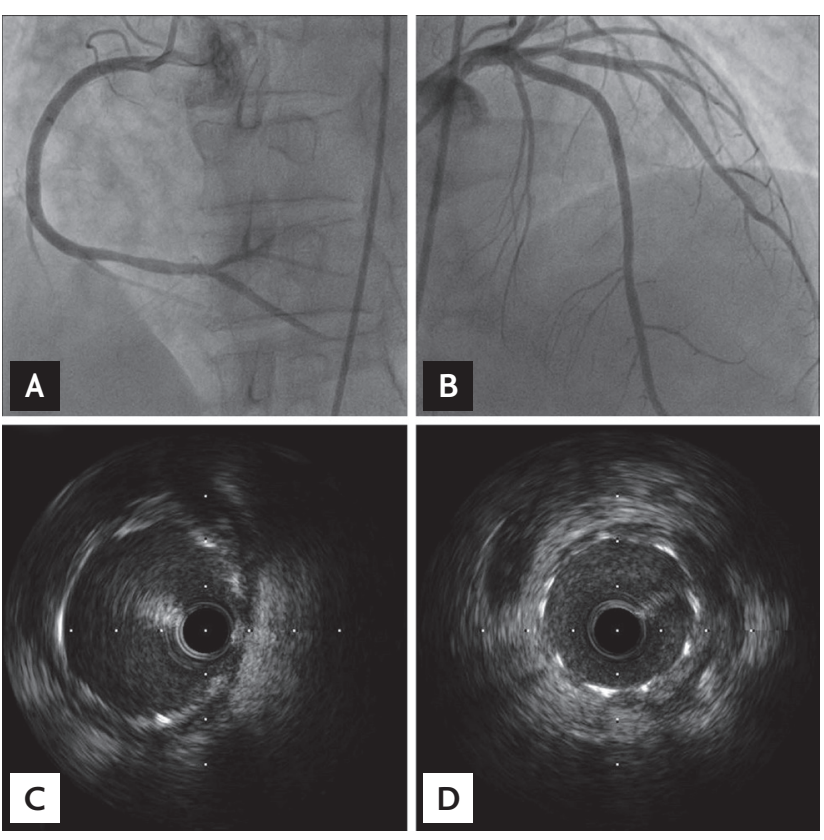

Figure 3. Successful percutaneous coronary intervention using everolimus-eluting stents (XienceXpedition, Abbott Vascular) in the p-m right coronary artery (RCA) and p-m left anterior descending artery (LAD). (A) A $4.0 \times 33 \mathrm{~mm}$ for proximal RCA, $4.0 \times 38 \mathrm{~mm}$ for middle RCA, (B) $4.0 \times 14 \mathrm{~mm}$ for proximal LAD and $3.5 \times 38 \mathrm{~mm}$ for middle LAD. $(\mathrm{C}, \mathrm{D})$ Subsequent intravascular ultrasound demonstrated a fully covered dissection lesion.

ral hematoma can be misinterpreted as atherosclerotic disease. Intracoronary imaging, such as IVUS and optical coherence tomography, can support the diagnosis.

The etiology and pathogenesis of SCAD remain poorly understood, although SCAD has been associated with pregnancy, the peripartum period, the use of oral contraceptives, intense physical exercise, emotional stress, 
and connective tissue disorders [4]. Of those who suffer from $\mathrm{SCAD}, 40 \%$ to $82 \%$ are women and the mean age is 40 to 43 years. A large registry-based study of elderly patients suggested that atherosclerosis was another cause of SCAD. However, atherosclerotic causes were less common than non-atherosclerotic causes. Our patient most likely had idiopathic SCAD because he did not have coronary risk factors and was an elderly male. We found no evidence of a connective tissue disorder or a history of trauma or drug abuse. There was no significant atherosclerosis, calcified lesion, or coronary artery anomaly such as FMD on IVUS.

The clinical presentation depends on the extent and severity of the dissection and the coronary artery involved. Presenting symptoms can range from angina to ST elevation myocardial infarction, ventricular fibrillation, and sudden cardiac death [2]. In our case, sudden ongoing chest pain, elevated cardiac enzymes, and nonST elevation myocardial infarction were diagnosed. The optimal treatment strategy for SCAD remains controversial and hinges on the patient's hemodynamics, coronary flow, dissection location, number of involved vessels, and clinical situation. Conservative treatment is preferred for stable patients with middle or distal local dissection if the lumen diameter is not limited by more than $50 \%$ and coronary blood flow is adequate [4]. However, patients with ongoing chest pain, ischemia, or ST elevation on ECG should undergo primary revascularization [5]. PCI is the best treatment in cases where the dissection is short and involves only one vessel. However, the technical success rate of PCI is only $65 \%$ in some series, with failures occurring because guidewires were passed into the false lumen, areas of dissection or hematoma were extended, or the intramural hematoma was displaced. Surgery is recommended in cases with left main artery dissection or multivessel dissections, such as our case. We performed PCI because of the persistent ongoing chest pain, elevated cardiac enzymes, and decreased EF and IVUS was used to determine the extent and location of the dissection. However, there is no consensus on the appropriate extent of stent coverage in terms of whether the proximal portion should be covered only or the entire dissection area, in our case. We covered the entire lesion with coronary stents, including the hematoma, to prevent propagation to the distal vessel. Further prospective randomized trials are needed to confirm the use of PCI.

Keywords: Multivessel coronary dissection; Spontaneous coronary dissection; Coronary artery dissection, spontaneous

\section{Conflict of interest}

No potential conflict of interest relevant to this article was reported.

\section{REFERENCES}

1. Basso C, Morgagni GL, Thiene G. Spontaneous coronary artery dissection: a neglected cause of acute myocardial ischaemia and sudden death. Heart 1996;75:451-454.

2. Alfonso F. Spontaneous coronary artery dissection: new insights from the tip of the iceberg? Circulation 2012;126:667-670.

3. Saw J, Ricci D, Starovoytov A, Fox R, Buller CE. Spontaneous coronary artery dissection: prevalence of predisposing conditions including fibromuscular dysplasia in a tertiary center cohort. JACC Cardiovasc Interv 2013;6:44-52.

4. Tweet MS, Hayes SN, Pitta SR, et al. Clinical features, management, and prognosis of spontaneous coronary artery dissection. Circulation 2012;126:579-588.

5. Shamloo BK, Chintala RS, Nasur A, et al. Spontaneous coronary artery dissection: aggressive vs. conservative therapy. J Invasive Cardiol 2010;22:222-228. 